STRUCTURAL BIOLOGY COMMUNICATIONS

ISSN 2053-230X

Received 15 September 2021

Accepted 21 November 2021

Edited by F. T. Tsai, Baylor College of Medicine, Houston, USA

Keywords: dynemicin; natural products; biosynthetic gene clusters; enediynes; polyketides; anthraquinone; $\beta$-barrel; unknown function; Micromonospora chersina ATCC53710.

PDB reference: DynF from Micromonospora chersina, 6ubl

Supporting information: this article has supporting information at journals.iucr.org/f

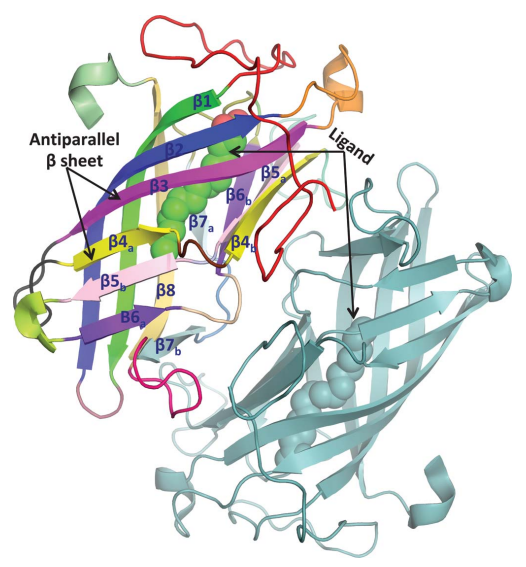
OPEN $\odot$ ACCESS

\section{The crystal structure of DynF from the dynemicin- biosynthesis pathway of Micromonospora chersina}

\author{
Abigael J. Kosgei, ${ }^{a}$ Mitchell D. Miller, ${ }^{a}$ Minakshi Bhardwaj, ${ }^{\mathrm{b}}$ Weijun $\mathrm{Xu},{ }^{\mathrm{a}}$ Jon S. \\ Thorson, ${ }^{\mathrm{b}, \mathrm{c}}$ Steven G. Van Lanen ${ }^{\mathrm{a}}$ and George N. Phillips Jr ${ }^{\mathrm{a}, \mathrm{d}}$ * \\ ${ }^{\mathbf{a}}$ Department of Biosciences, Rice University, Houston, TX 77251, USA, ${ }^{\mathbf{b}}$ Department of Pharmaceutical Sciences, \\ College of Pharmacy, University of Kentucky, Lexington, KY 40506, USA, ${ }^{\mathrm{c} C e n t e r}$ for Pharmaceutical Research and \\ Innovation, College of Pharmacy, University of Kentucky, Lexington, KY 40506, USA, and dDepartment of Chemistry, \\ Rice University, Houston, TX 77251, USA. *Correspondence e-mail: georgep@rice.edu
}

Dynemicin is an enediyne natural product from Micromonospora chersina ATCC53710. Access to the biosynthetic gene cluster of dynemicin has enabled the in vitro study of gene products within the cluster to decipher their roles in assembling this unique molecule. This paper reports the crystal structure of DynF, the gene product of one of the genes within the biosynthetic gene cluster of dynemicin. DynF is revealed to be a dimeric eight-stranded $\beta$-barrel structure with palmitic acid bound within a cavity. The presence of palmitic acid suggests that DynF may be involved in binding the precursor polyene heptaene, which is central to the synthesis of the ten-membered ring of the enediyne core.

\section{Introduction}

Dynemicin $\mathrm{A}$ is an anthraquinone-fused ten-membered enediyne and a metabolite of Micromonospora chersina ATCC53710. Like many other enediynes, dynemicin has a novel molecular architecture, a notable mode of action and potent cytotoxicity (Thorson et al., 2000; Van Lanen \& Shen, 2008). Dynemicin comprises an enediyne core, which is common to all enediynes, and peripheral moieties (Fig. 1), which give the enediynes structural diversity. The main ring of the dynemicin core contains two acetylenic groups conjugated to a double bond in a ten-membered carbocyle. The enediyne core structural unit (termed the warhead) is central to the fundamental mechanism of action. The anthraquinone group on the peripheral moeity of dynemicin intercalates into the minor groove of DNA to confer binding specificity (Sugiura $e t$ al., 1990).

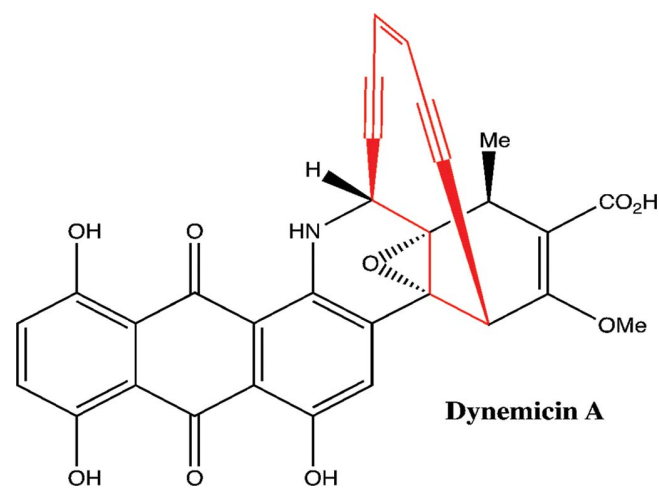

Figure 1

Dynemicin A has a unique molecular architecture comprising an enediyne core (red) and peripheral moieties (black). The enediyne core is the key to the cytotoxicity of dynemicin. 
In the presence of NADPH or thiol, the quinone is reduced to hydroquinone, leading to opening of the epoxide (Avendaño \& Menéndez, 2015), which triggers the activation of the enediyne core. Dynemicin then undergoes a Bergman cycloaromatization to form a reactive 1,4-dehydrobenzene biradical that is capable of abstracting $\mathrm{H}$ atoms from the DNA backbone, causing single- or double-stranded DNA scission. The high reactivity of enediynes with DNA makes them quite toxic. In natural environments, some microorganisms producing enediynes protect themselves from their cytotoxic action by a self-resistance mechanism that uses a self-sacrificing protein (Biggins et al., 2003; Elshahawi et al., 2014) or specialized ABC transporters to export the toxin (Beis \& Rebuffat, 2019). In cancer therapy, the toxicity of enediynes has been exploited by conjugating the molecules to tumorspecific monoclonal antibodies in polymer-assisted delivery systems to prevent premature cytotoxic effects on other important cells in the body (Abe \& Otsuki, 2002; Shah, 2017).

Dynemicin can be produced by organic synthesis or in M. chersina. The total chemical synthesis of enediynes is challenging, but has been achieved for dynemicin (Nicolaou et al., 1991, 1993; Shair et al., 1996; Myers et al., 1995). The cloning, sequencing and identification of the biosynthetic gene clusters of dynemicin has paved the way for in vitro studies of these biosynthetic enzymes (Gao \& Thorson, 2008). The putative biosynthesis of dynemicin is correspondingly complex: the $M$. chersina whole genome sequence analysis revealed 60 open reading frames (ORFs) within the biosynthetic gene cluster related to dynemicin production. Using homology to other characterized genomes, these genes have been annotated to suggest functions such as biosynthesis of the enediyne core, maturation and regulation, while there are also those with as yet unknown functions (Gao \& Thorson, 2008). The gene cluster contains a common iterative type I polyketide synthase (PKSE) that is essential for the biosynthesis of a precursor polyene (heptaene) which, in the presence of other accessory enzymes, forms the ten-membered enediyne core of dynemicin (Liu et al., 2003). Evidence has accumulated that the PKSE product is also the precursor of the anthraquinone, and the biosynthesis of this moiety proceeds through a $\delta$-thiolactone iodoanthracene intermediate (Cohen \& Townsend, 2018). While some insight into the conversion of the iodoanthracene to the anthraquinone has recently been reported upon examination of the biosynthesis of sungeidines (Ma et al., 2021), which, like dynemicin, are anthraquinone-fused ten-membered enediynes, the steps leading to the iodoanthracene in addition to the enediyne core remain mostly unknown.

This study targets one of several proteins of unknown function encoded by a gene within the biosynthetic gene cluster of dynemicin, herein named DynF. The gene encoding DynF was originally annotated orf15 (Gao \& Thorson, 2008) and was deposited as ORF18 with unknown function (GenBank accession No. ACB47058.1). The DynF ortholog Sgd15 was very recently demonstrated by gene inactivation to be essential for sungeidine biosynthesis, and no intermediates, including the iodoanthracene, were detected (Ma et al., 2021), suggesting that DynF and Sgd15 are involved in the early steps of processing the PKSE product to either the enediyne core or iodoanthracene, or potentially both. Here, we report the structural characterization of DynF using X-ray crystallography as an initial step towards deciphering its role in the biosynthesis of this unique anthraquinone-fused enediyne.

\section{Materials and methods}

\subsection{Macromolecule production}

A construct for DynF from M. chersina ATCC53710 with a cleavable $6 \times$ His expression and purification tag on the $\mathrm{N}$-terminus was codon-optimized for production in Escherichia coli. It was ordered as a synthetic gBlock from Integrated DNA Technologies (IDT, Coralville, Iowa, USA). Amplification of the gene was performed by polymerase chain reaction (PCR) using Q5 High-Fidelity $2 \times$ Master Mix (NEB, Westwood, Massachusetts, USA). Gibson assembly was used to clone the gene into a pNIC28-Bsa4 vector (Savitsky et al., 2010; Addgene, Watertown, Masachusetts, USA). It was then transformed into DH5 $\alpha$ competent $E$. coli cells (Invitrogen, Carlsbad, California, USA). Sanger sequencing (Genewiz, South Plainfield, New Jersey, USA) was used to verify the sequence of the construct.

The DynF_pNIC28-Bsa4 plasmid was transformed into competent E. coli BL21 (DE3) expression cells (Agilent Technologies, Cedar Creek, Texas, USA). $5 \mu$ l of the transformed cells were then grown overnight on Luria-Bertani (LB) agar plates containing $50 \mu \mathrm{g} \mathrm{ml}^{-1}$ kanamycin antibiotic. From a single colony, a seed culture of $50 \mathrm{ml}$ was grown in LB medium supplemented with $50 \mu \mathrm{g} \mathrm{ml}^{-1}$ kanamycin to select for expression in the cells. DynF was expressed in BL21 (DE3) competent $E$. coli cells and was most soluble when induced with $0.5 \mathrm{~m} M$ isopropyl $\beta$-D-1-thiogalactopyranoside (IPTG) and cultured at $291 \mathrm{~K}$ after induction. To obtain suitable quantities of DynF protein for crystallization experiments, 11 culture was grown from a $50 \mathrm{ml}$ overnight seed culture until an $\mathrm{OD}_{600}$ of $\sim 0.6-0.8$ was reached. The cells were then induced with $0.5 \mathrm{~m} M$ IPTG and cultured at $291 \mathrm{~K}$ for $17 \mathrm{~h}$. These cells were harvested by centrifugation at $5000 \mathrm{rev} \mathrm{min}^{-1}$ for $40 \mathrm{~min}$.

The cell pellet was resuspended using a lysis buffer consisting of $10 \%$ glycerol, $20 \mathrm{~m} M$ imidazole, $50 \mathrm{mM}$ 4-(2hydroxyethyl)-1-piperazineethanesulfonic acid (HEPES) $\mathrm{pH}$ 8.0, $500 \mathrm{~m} M \quad \mathrm{NaCl}, 1 \mathrm{~m} M$ tris(2-carboxyethyl)phosphine hydrochloride (TCEP). The cells were then lysed by sonication for $4 \mathrm{~min} 30 \mathrm{~s}$ pulse on and $2 \mathrm{~min}$ pulse off with $50 \%$ amplitude (Qsonica Q700 sonicator, Newton, Connecticut, USA). The lysed cells were centrifuged at $20000 \mathrm{rev} \mathrm{min}^{-1}$ for $45 \mathrm{~min}$. Immobilized metal-affinity chromatography (IMAC) with Ni-NTA resin (Qiagen, Hilden, Germany) was used to purify the protein. The protein was eluted from the nickel column using a buffer consisting of $10 \%$ glycerol, $500 \mathrm{mM}$ imidazole, $25 \mathrm{~m} M$ HEPES pH 8.0, $500 \mathrm{~m} M \mathrm{NaCl}, 1 \mathrm{~m} M$ TCEP. Sodium dodecyl sulfate-polyacrylamide gel electrophoresis (SDS-PAGE) was used to confirm the production of DynF protein with an expected size of $22.6 \mathrm{kDa}$. The protein was 
concentrated and buffer-exchanged into Tobacco etch virus (TEV) protease buffer (25 $\mathrm{m} M$ Tris $\mathrm{pH} 8.0,150 \mathrm{~m} M \mathrm{NaCl}$, $1 \mathrm{~m} M$ DTT) using an Amicon ultracentrifugal filter (10 000 molecular-weight cutoff, Merck KGaA, Darmstadt, Germany). The protein concentration was obtained using a $\mathrm{UV}-\mathrm{Vis}$ spectrophotometer with an extinction coefficient of 1.191, and was confirmed using a NanoDrop. The histidine tag at the N-terminus of the DynF protein was cleaved from the protein using His-tagged TEV protease. Subtractive nickel column purification was performed to remove the uncleaved protein and protease. DynF was run on a size-calibrated HiLoad 16/600 Superdex 200 pg (GE Healthcare, Uppsala, Sweden) size-exclusion column (SEC) to further purify it and analyze its oligomeric state (Supplementary Fig. S1). Macromolecule-production information is summarized in Table 1.

\subsection{Crystallization}

DynF was concentrated to $20 \mathrm{mg} \mathrm{ml}^{-1}$ and subjected to high-throughput crystallization screening. A Mosquito LCP robot (SPT Labtech) was used with commercially available screens, including Wizard 1 and 2 and Wizard 3 and 4 (Rikagu Reagents, Bainbridge Island, Washington, USA), PEGRx and Crystal Screen HT (Hampton Research, Aliso Viejo, California, USA). Screens were set up via the sitting-drop vapordiffusion method in MRC 2 96-well crystallization plates (Hampton Research, Aliso Viejo, California, USA) with drops consisting of $200 \mathrm{nl}$ protein solution $\left(20 \mathrm{mg} \mathrm{ml}^{-1}\right)$ mixed with $200 \mathrm{nl}$ reservoir solution. Initial crystal hits (Fig. 2a) were observed in two wells of the Wizard 3 and 4 crystallization screen [C1, 10\%(w/v) PEG 1000, 10\%(w/v) PEG 8000; F10, $25 \%(w / v)$ PEG 1500, 0.1 M PCB buffer $\mathrm{pH}$ 7.0]. Optimization for larger crystals from well F10 of the Wizard 3 and 4 crystallization screen was achieved by increasing the protein: precipitant ratio (300:200 nl) in the initial drop (Fig. 2b). Potassium iodide (KI) derivatives were prepared by soaking native DynF crystals for $30 \mathrm{~s}$ in $0.5 \mathrm{~m} M$ KI dissolved in the reservoir solution. Native and KI-soaked crystals were looped

Table 1

Macromolecule-production information.

Source organism

DNA source

Insert forward primer $\dagger$

Insert reverse primer

Vector forward primer

Vector reverse primer

Expression vector $\$$

Expression host

Complete amino-acid sequence of the construct produced\$

\begin{abstract}
Micromonospora chersina
E. coli codon-optimized synthesis (gBlock) from Genewiz

5'-agaacctgtacttccaatccATGTCGA CTAAATCTGTATT-3'

5'-ACCAGACCAGATGTGAacgg tctccag taaaggtgg- $3^{\prime}$

5'-TTAAACCAGACCAGATGTGAacgg tct Ccagtaaaggtg-3'

5'-agaacctgtacttccaatcCATGTCGA CTAAATCTGTATT-3

pNIC28-Bsa4

Escherichia coli BL21 (DE3)

mgsshhhhhhenlyfq/sMSTKSVLFGRPV QTEGVPNVYAGAPVVPWTPPEPGIDNLG INS IDTFAVPGVGEYTVAFDGWVRVVRS PSTSGEWADAEVYTNLIEMKMVGECEEL GKITVTLNPDCLSAGQIRTPFDPYAGEG PSAKACRMAVGAIFDMPKLGLKLMNREP I ILTIDDVRS I PPAGAPGKGQIYRMMPL LDVNDPDGQPVAYLTSLRFNMGGYLKPD QM
\end{abstract}

$\dagger$ The primers contained overhangs for cloning, which are represented as lower case letters. \$ Plasmid pNIC28-Bsa4 was a gift from Opher Gileadi (Addgene plasmid \#26103). $\$$ The expression and purification tag is shown in lower case with the TEV recognition site underlined and the cut site indicated with a slash.

using Mounted CryoLoops (Hampton Research, Aliso Viejo, California, USA), cryocooled in liquid nitrogen and shipped to the Advanced Photon Source synchrotron facility at Argonne National Laboratory. Crystallization information is summarized in Table 2.

\subsection{Data collection and processing}

X-ray diffraction data were collected remotely at wavelengths of 1.0332 and $1.823 \AA$ for the native crystals and the heavy-atom derivative crystals, respectively. The native and iodide-soaked crystals diffracted to resolutions of 1.55 and $2.38 \AA$, respectively. The DynF diffraction data were indexed in the orthorhombic space group $C 222_{1}$, with unit-cell para-

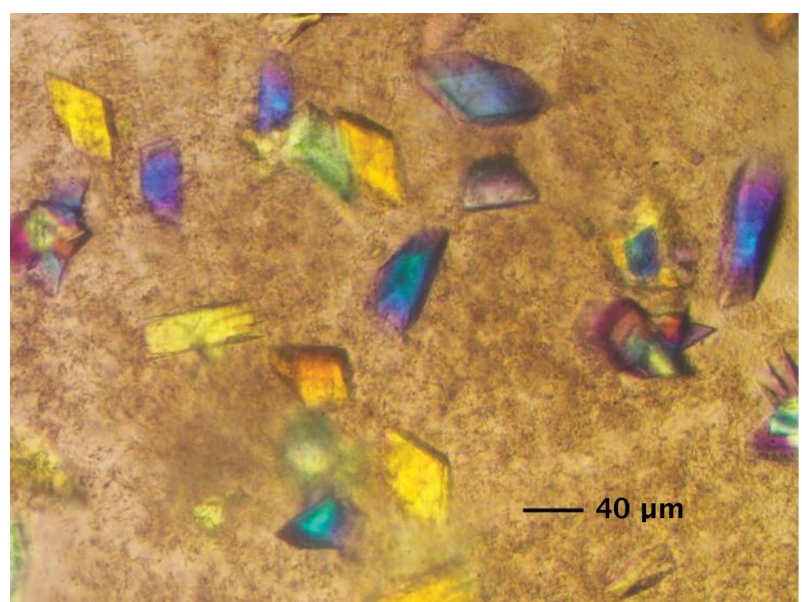

(a)

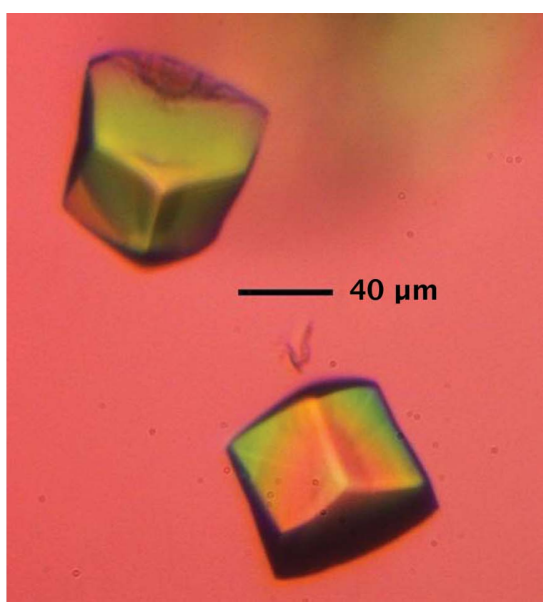

(b)

Figure 2

DynF crystals as viewed under an Olympus BHTP polarizing microscope equipped with an Olympus OM-D E-M5 digital camera. (a) Initial crystal hits observed at a 1:1 ratio of protein to precipitant. (b) Larger crystals were obtained by increasing the amount of protein in the crystallization wells. 
Table 2

Crystallization.

\begin{tabular}{ll}
\hline Method & Vapor diffusion, sitting drop \\
Plate type & MRC 2 (96-well crystallization plate) \\
Temperature (K) & 293 \\
Protein concentration $\left(\mathrm{mg} \mathrm{ml}^{-1}\right)$ & 20 \\
Buffer composition of protein & $0.025 M$ Tris pH 8.0, 0.15 M NaCl, $1 \mathrm{~m} M$ \\
solution (crystallization buffer) & $\mathrm{DTT}$ \\
Composition of reservoir solution & $25 \%(w / v)$ PEG 1500, 0.1 M PCB buffer \\
& $\mathrm{pH} \mathrm{7.0}$ \\
Volume and ratio of drop $\dagger$ & $300 \mathrm{nl}(1.5: 1)$ \\
Volume of reservoir $(\mu \mathrm{l})$ & 50
\end{tabular}

$\dagger$ The initial screening for crystals was performed using a 1:1 ratio of protein to reservoir solution.

Table 3

Data collection and processing.

Values in parentheses are for the outer shell.

\begin{tabular}{|c|c|c|}
\hline & Native & KI soaked \\
\hline Diffraction source & $\begin{array}{l}\text { Beamline 23-ID-B, } \\
\text { APS }\end{array}$ & $\begin{array}{l}\text { Beamline 23-ID-B, } \\
\text { APS }\end{array}$ \\
\hline Wavelength $(\AA ̊)$ & 1.0332 & 1.8233 \\
\hline Temperature (K) & 100 & 100 \\
\hline Detector & $\begin{array}{l}\text { Dectris EIGER X } \\
16 \mathrm{M}\end{array}$ & $\begin{array}{l}\text { Dectris EIGER X } \\
16 \mathrm{M}\end{array}$ \\
\hline $\begin{array}{l}\text { Crystal-to-detector distance } \\
(\mathrm{mm})\end{array}$ & 219.1 & 199.4 \\
\hline Rotation range per image $\left({ }^{\circ}\right)$ & 0.2 & 0.2 \\
\hline Total rotation range $\left({ }^{\circ}\right)$ & 180 & 360 \\
\hline Exposure time per image (s) & 0.2 & 0.2 \\
\hline Space group & $C 222_{1}$ & $C 222_{1}$ \\
\hline$a, b, c(\AA)$ & $59.83,116.13,129.88$ & $59.93,116.08,131.03$ \\
\hline Mosaicity $\left({ }^{\circ}\right)$ & 0.14 & 0.15 \\
\hline Resolution range $(\AA)$ & $34.7-1.50(1.59-1.50)$ & $49.3-2.38(2.53-2.40)$ \\
\hline Total No. of reflections & 440004 (21559) & 198086 (8709) \\
\hline No. of unique reflections & $70922(5926)$ & $17513(1990)$ \\
\hline Completeness (\%) & $97.6(87.1)$ & $93.7(66.8)$ \\
\hline Multiplicity & $6.2(3.9)$ & $11.3(4.4)$ \\
\hline$\langle I / \sigma(I)\rangle$ & $14.1(0.63) \dagger$ & $14.1(1.8) \neq$ \\
\hline$R_{\text {meas }}$ & $0.061(1.90)$ & $0.120(0.47)$ \\
\hline $\mathrm{CC}_{1 / 2}$ & $1.00(0.37)$ & $0.998(0.88)$ \\
\hline $\begin{array}{l}\text { Overall } B \text { factor from Wilson } \\
\text { plot }\left(\AA^{2}\right)\end{array}$ & 32 & 50 \\
\hline
\end{tabular}

$\dagger\langle I / \sigma(I)\rangle$ falls below 2.0 at $1.67 \AA$ resolution. $\ddagger\langle I / \sigma(I)\rangle$ falls below 2.0 at $2.47 \AA$ resolution.

meters $a=60, b=65, c=131 \AA$. The diffraction data were integrated and scaled using XDS (Kabsch, 2010a,b). Datacollection and processing statistics are summarized in Table 3.

\subsection{Structure solution and refinement}

The SAD (single-wavelength anomalous diffraction) and SIRAS (single isomorphous replacement with anomalous scattering) methods were used to phase the structure of DynF. Scaling, substructure solution and phasing were accomplished with SHELXC/D/E (Sheldrick, 2010) using the HKL2MAP graphical interface (Pape \& Schneider, 2004). Automated protein tracing with $A R P / w A R P$ (Langer et al., 2008) was used on both the SAD and the SIRAS maps to build 418 of 422 residues.

Refinement and model building were performed using phenix.refine (Afonine et al., 2012) and Coot (Emsley et al., 2010). The structure was visualized using a collaborative 3D
Table 4

Structure solution and refinement.

Values in parentheses are for the outer shell.

\begin{tabular}{ll}
\hline PDB entry & $6 \mathrm{ubl}$ \\
Resolution range $(\AA)$ & $34.7-1.50(1.52-1.50)$ \\
Completeness $(\%)$ & 97.3 \\
No. of reflections, working set & $70715(2000)$ \\
No. of reflections, test set & $3518(117)$ \\
Final $R_{\text {work }}$ & $0.161(0.405)$ \\
Final $R_{\text {free }}$ & $0.185(0.362)$ \\
No. of non-H atoms & \\
$\quad$ Protein & 3287 \\
$\quad$ Ligand & 3 \\
$\quad$ Solvent & 422 \\
$\quad$ Total & 374 \\
Ligand RSC & \\
$\quad$ Palmitic acid A & 0.94 \\
$\quad$ Palmitic acid B & 0.91 \\
R.m.s. deviations & \\
$\quad$ Bonds $(\AA)$ & 0.007 \\
Angles $\left({ }^{\circ}\right)$ & 1.0 \\
Average $B$ factors $\left(\AA^{2}\right)$ & 32 \\
$\quad$ Protein & 31 \\
Ligand & 46 \\
Water & 41 \\
Ramachandran plot & \\
$\quad$ Most favored $(\%)$ & 99.3 \\
Allowed outliers $(\%)$ & 0.0 \\
\hline
\end{tabular}

graphics system (Yennamalli et al., 2014). The software applications used in this project were compiled and configured by SBGrid (Morin et al., 2013). The structure of DynF and its corresponding diffraction data have been deposited in the worldwide Protein Data Bank (wwPDB; Berman et al., 2003) as entry 6ubl. Refinement statistics are summarized in Table 4.

\subsection{Ligand determination using gas chromatography-mass spectrometry (GC-MS)}

Acetonitrile $(0.5 \mathrm{ml})$ was added to $0.5 \mathrm{ml}$ purified DynF protein $\left(40 \mathrm{mg} \mathrm{ml}^{-1}\right)$ and rotated at room temperature for $2 \mathrm{~h}$. The mixture was centrifuged using a protein concentrator (3000 molecular-weight cutoff; Millipore, Cork, Ireland) at $4000 \mathrm{rev} \mathrm{min}^{-1}$ for $20 \mathrm{~min}$, and the acetonitrile extract $(100 \mu \mathrm{l})$ was filtered to remove any remaining insoluble debris. Gas chromatography-mass spectrometry (GC-MS) analysis was performed using an Agilent $7890 \mathrm{GC}$ with a $30 \mathrm{~m} \times 250 \mu \mathrm{m} \times$ $0.25 \mathrm{~mm}$ HP-5ms UI capillary column and an Agilent G7081B MSD. Helium was the carrier gas at a flow rate of $1 \mathrm{ml} \mathrm{min}^{-1}$. The temperatures of the injector, the interface and the liner were $523 \mathrm{~K}$. Injections of $5 \mu \mathrm{l}$ were performed in splitless mode. The column temperature was initially $343 \mathrm{~K}$ and was held for $2 \mathrm{~min}$, increased to $513 \mathrm{~K}$ at $277 \mathrm{~K} \mathrm{~min}^{-1}$ and run for a total run time of $59.5 \mathrm{~min}$. For GC-MS detection, an electronimpact ionization system was used. The mass range was set to $50-500 \mathrm{~m} / \mathrm{z}$ with a filament delay of $4 \mathrm{~min}$. The sampleinjection volume was $1 \mu \mathrm{l}$.

\subsection{Co-expression of DynF with SgcE and DynE7}

The genes encoding the C-1027 polyketide synthase SgcE and dynemicin thioesterase DynE7 were cloned into pETDuet using standard procedures. The gene encoding DynF was cloned into pDB.His.MBP. All genes were sequenced to 
confirm their identity. Both pETDuet and pDB.His.MBP plasmids with the respective genes were co-transformed into E. coli BL21 (DE3) cells. The transformed cells were plated on LB agar supplemented with $50 \mu \mathrm{g} \mathrm{ml}^{-1}$ kanamycin and $50 \mu \mathrm{g} \mathrm{ml}^{-1}$ carbenicillin. After overnight incubation at $310 \mathrm{~K}$, a single colony was used to inoculate $5 \mathrm{ml} \mathrm{LB}$ medium supplemented with $50 \mu \mathrm{g} \mathrm{ml}^{-1}$ kanamycin and $50 \mu \mathrm{g} \mathrm{m}^{-1}$ carbenicillin. Incubation was performed overnight at $310 \mathrm{~K}$ with shaking at $250 \mathrm{rev} \mathrm{min}^{-1}$. The culture $(0.5 \mathrm{ml})$ was transferred into $50 \mathrm{ml}$ fresh LB supplemented with $50 \mu \mathrm{g} \mathrm{ml}^{-1}$ kanamycin and $50 \mu \mathrm{g} \mathrm{ml}^{-1}$ carbenicillin and incubated at $291 \mathrm{~K}$ and $250 \mathrm{rev} \mathrm{min}^{-1}$. When the optical density at $600 \mathrm{~nm}$

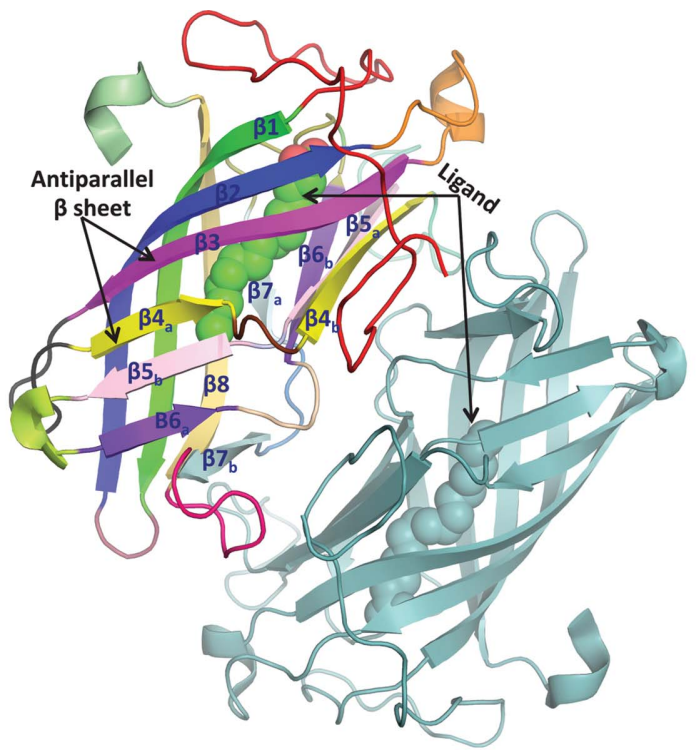

(a)

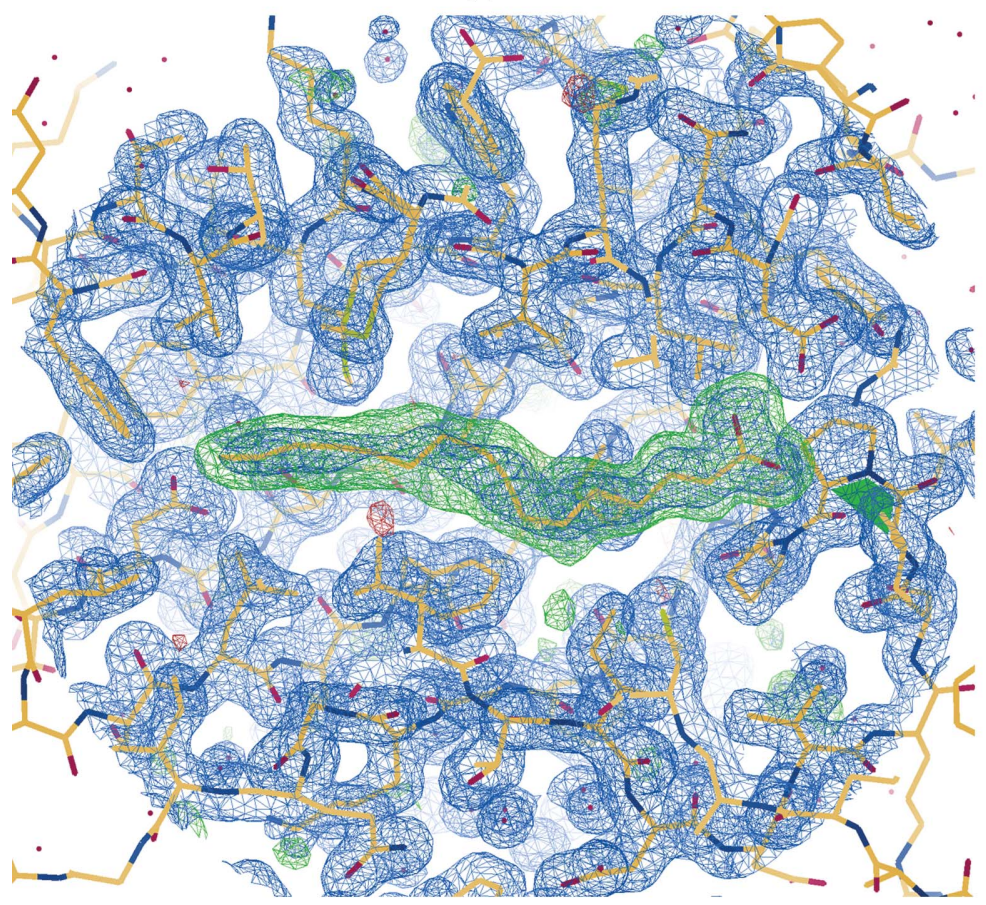

(c)

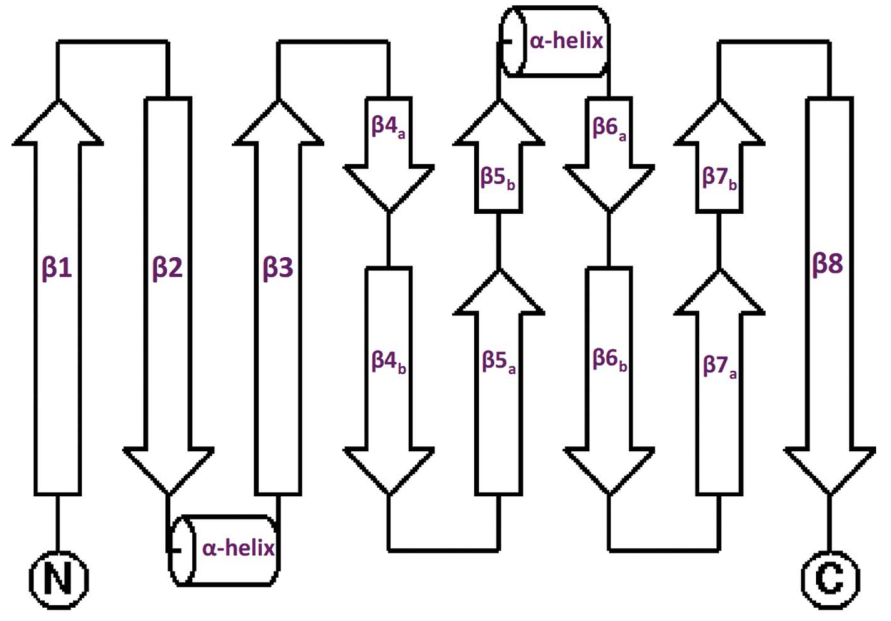

(b)

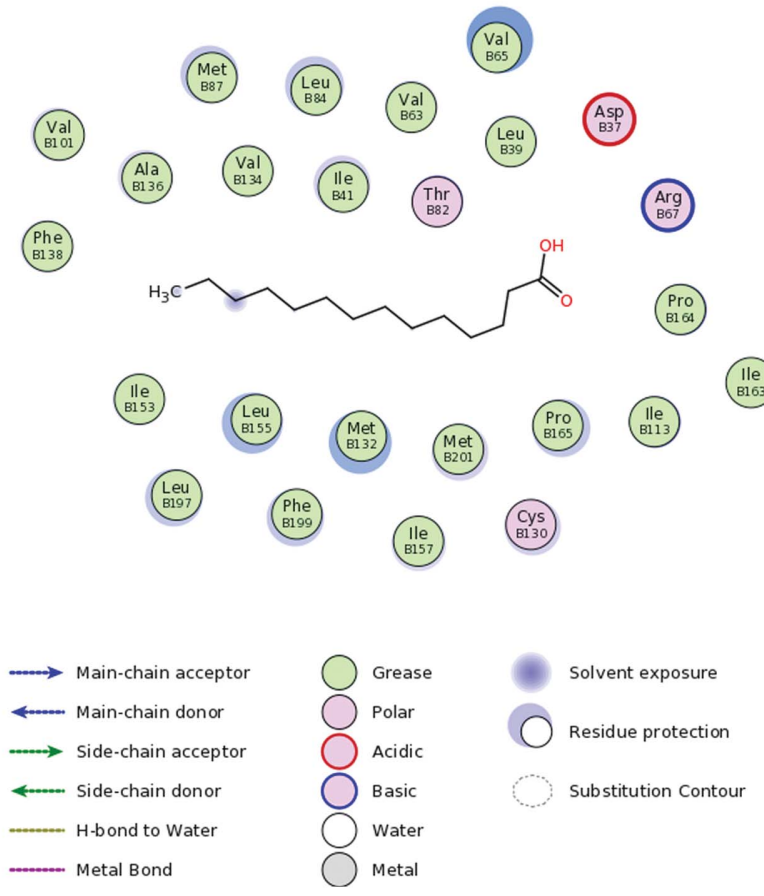

(d)

Figure 3

Structure of DynF. (a) A ribbon diagram of the crystal structure of DynF (PDB entry 6ubl) viewed down its noncrystallogaphic twofold axis. The ligand is represented as spheres embedded in the DynF $\beta$-barrels. Each protomer of DynF comprises an eight-stranded antiparallel $\beta$-barrel connected by loops and two helical segments. Strands 4, 5, 6 and 7 are interrupted by dimerization interactions. This figure was generated in $P y M O L$ (version 2.4.1; Schrödinger). (b) A topology diagram of DynF generated using TopDraw (Bond, 2003) shows that each strand is connected to its next neighbor, as is typical of most eight-stranded antiparallel $\beta$-barrel structures. (c) The final $2 m F_{\mathrm{o}}-m F_{\mathrm{c}}$ map (contoured at 1 r.m.s.d) in blue with \pm 3 r.m.s.d. contours of the polder omit map in green/red represented in Coot. The green positive difference density clearly shows the location of the bound ligand (palmitic acid) that was omitted from the model phases for the polder map calculation. (d) The ligand in the DynF structure is surrounded by hydrophobic aminoacid groups, except for residues that interact with the carboxylic group at one end of the palmitic acid. This figure was generated with the FLEV (Flatland Ligand Environment View) tool in Coot. 
reached 0.5-0.6, expression was induced with sterile IPTG solution (final concentration $0.1 \mathrm{mM}$ ) and incubated at $291 \mathrm{~K}$ and $250 \mathrm{rev} \mathrm{min}^{-1}$ for an additional $36 \mathrm{~h}$. The cells were centrifuged at $4000 \mathrm{rev} \mathrm{min}^{-1}$ at $277 \mathrm{~K}$ for $30 \mathrm{~min}$, and the wet pellet was washed once with $30 \mathrm{ml}$ phosphate buffer $\mathrm{pH}$ 7.2. The pellet was resuspended in $30 \mathrm{ml}$ phosphate buffer $\mathrm{pH} 7.2$ and sonicated for 2 min with $10 \mathrm{~s}$ pulse on and $50 \mathrm{~s}$ pulse off (with $40 \%$ amplitude) on ice. The lysate was purified using nickel resin and then with amylose resin to purify the MBPtagged DynF. The sample was extracted with acetonitrile and analyzed by GC-MS as described above.

\section{Results and discussion}

The structure of DynF was determined to a resolution of $1.50 \AA$ with a final $R_{\text {work }}$ and $R_{\text {free }}$ of 0.16 and 0.19 , respectively. The DynF crystal has two molecules in its asymmetric unit (Fig. 3a). An analysis of the crystal contacts with PISA (Krissinel \& Henrick, 2007) predicts that DynF forms a stable dimer. Size-exclusion chromatography (SEC) showed that DynF could exist in a higher oligomeric state in solution (Supplementary Fig. S1).

DynF is comprised of antiparallel $\beta$-sheets that are connected by short loops and helical segments to form eightstranded antiparallel $\beta$-barrels (Supplementary Fig. S2). The $\beta$-strands of the DynF structure are labeled 1, 2, 3, 4a/4b, 5a/ $5 \mathrm{~b}, 6 \mathrm{a} / 6 \mathrm{~b}, 7 \mathrm{a} / 7 \mathrm{~b}$ and 8 . Two short helical segments connect $\beta$-strands $2 / 3$ and $5 \mathrm{~b} / 6 \mathrm{a}$. The topology of DynF shows that each strand is directly linked to its neighbor, which is typical of most topologies of eight-stranded antiparallel $\beta$-barrels (Fig. $3 b$ ) such as outer membrane proteins, avidins, fatty-acid binding proteins and lysozyme inhibitors (Geerds et al., 2014). Deviating from a canonical eight-stranded, up-down $\beta$-barrel (Lalonde et al., 1994), we observed short loops inserted within strands 4, 5, 6 and 7 (Figs. $3 a$ and $3 b$ ). Some of the loop residues within strands 6 and 7 are located at the dimer interface and thus may be involved in stabilization of the dimer (Supplementary Fig. S3, Supplementary Tables S1 and S2).

Comparative analysis of protein structures can help to decipher their functions. The DynF structure was compared with other structures in the Protein Data Bank (PDB) using the DALI server (Holm, 2020). DynF did not have significant structural similarities to previous structures $(Z$-score of $<10$; structural identity of $<15 \%$; Supplementary Table S3, Supplementary Fig. S4), suggesting that DynF represents a novel fold, thus lacking precedents for providing a known mechanism.

The electron-density map of DynF revealed an elongated ligand embedded within the $\beta$-barrel (Fig. $3 c$ ). The size and shape of the ligand, as well as its binding environment, are suggestive of a 16-carbon fatty acid, which was confirmed by gas chromatography-mass spectrometry (GC-MS) as palmitic acid (Supplementary Fig. S5). Palmitic acid is the most abundant fatty acid expressed in E. coli cells (Cronan \& Thomas, 2009). We hypothesize that the ligand co-purified with the protein. The surface representation of DynF in
PyMOL reveals that the binding cavity of the ligand is closed and may thus require the channel within the ligand-binding site to open and close in order to allow the ligand to enter and exit.

The hydrophobicity of the side chains of the residues in the ligand-binding cavity (Fig. $3 d$ ) and the presence of palmitic acid suggest that DynF may be involved in the binding or processing of the PKSE product or an early biosynthetic intermediate, which is consistent with the results from the inactivation of $\operatorname{sgd15}$ (Ma et al., 2021). A linear conjugated heptaene has previously been observed upon heterologous co-expression of the genes encoding PKSE and a type II thioesterase in E. coli (Annaval et al., 2017; Smanski et al., 2011), and was hypothesized to potentially serve as the DynF ligand or substrate. To test this, DynF was co-expressed with a C-1027 polyketide synthase, SgcE, and a dynemicin thioestarase, DynE7. However, GC-MS analysis of the ligand following purification of DynF after co-expression of the three genes gave an identical profile predominantly containing palmitic acid. Thus, the role of DynF has not directly been confirmed. Nonetheless, the structural data now provide insight into its potential role in binding the precursor polyene and provide an opportunity to further explore this role via in vitro assays.

\section{Acknowledgements}

This work used research resources provided by the Center of Biomedical Research Excellence (COBRE) in Pharmaceutical Research and Innovation (CPRI; NIH P20 GM130456), the University of Kentucky College of Pharmacy and the National Center for Advancing Translational Sciences (UL1TR000117 and UL1TR001998). This research used resources of the Advanced Photon Source, a US Department of Energy (DOE) Office of Science User Facility operated for the DOE Office of Science by Argonne National Laboratory under Contract No. DE-AC02-06CH11357. GM/CA@APS has been funded by the National Cancer Institute (ACB-12002) and the National Institute of General Medical Sciences (AGM-12006, P30GM138396). This content is solely the responsibility of the authors and does not necessarily represent the official views of the National institutes of Health or the National Science Foundation.

\section{Funding information}

This research was funded by National Institutes of Health grant R01 GM115261 (JST and GNP), National Cancer Institute grant R01 CA217255 (JST, SGVL and GNP) and National Science Foundation, BioXFEL Science and Technology Center grant No. 1231306 (GNP).

\section{References}

Abe, S. \& Otsuki, M. (2002). Curr. Med. Chem. Anticancer Agents, 2 , 715-726.

Afonine, P. V., Grosse-Kunstleve, R. W., Echols, N., Headd, J. J., Moriarty, N. W., Mustyakimov, M., Terwilliger, T. C., Urzhumtsev, A., Zwart, P. H. \& Adams, P. D. (2012). Acta Cryst. D68, 352-367. 
Annaval, T., Rudolf, J., Chang, C., Lohman, J., Kim, Y., Bigelow, L., Jedrzejczak, R., Babnigg, G., Joachimiak, A., Phillips, G. \& Shen, B. (2017). ACS Omega, 2, 5159-5169.

Avendaño, C. \& Menéndez, J. C. (2015). Medicinal Chemistry of Anticancer Drugs, 2nd ed, pp. 133-195. Amsterdam: Elsevier.

Beis, K. \& Rebuffat, S. (2019). Res. Microbiol. 170, 399-406.

Berman, H. M., Henrick, K. \& Nakamura, H. (2003). Nat. Struct. Mol. Biol. 10, 980.

Biggins, J., Onwueme, K. C. \& Thorson, J. S. (2003). Science, 301, $1537-1541$.

Bond, C. S. (2003). Bioinformatics, 19, 311-312.

Cohen, D. \& Townsend, C. (2018). Angew. Chem. 130, 5752-5756.

Cronan, J. \& Thomas, J. (2009). Methods Enzymol. 459, 395-433.

Elshahawi, S. I., Ramelot, T. A., Seetharaman, J., Chen, J., Singh, S., Yang, Y., Pederson, K., Kharel, M. K., Xiao, R., Lew, S., Yennamalli, R. M., Miller, M. D., Wang, F., Tong, L., Montelione, G. T., Kennedy, M. A., Bingman, C. A., Zhu, H., Phillips, G. N. Jr \& Thorson, J. S. (2014). ACS Chem. Biol. 9, 2347-2358.

Emsley, P., Lohkamp, B., Scott, W. G. \& Cowtan, K. (2010). Acta Cryst. D66, 486-501.

Gao, Q. \& Thorson, J. (2008). FEMS Microb. Lett. 282, 105-114.

Geerds, C., Wohlmann, J., Haas, A. \& Niemann, H. H. (2014). Acta Cryst. F70, 866-871.

Holm, L. (2020). Methods Mol. Biol. 2112, $29-42$.

Kabsch, W. (2010a). Acta Cryst. D66, 125-132.

Kabsch, W. (2010b). Acta Cryst. D66, 133-144.

Krissinel, E. \& Henrick, K. (2007). J. Mol. Biol. 372, 774-797.

Lalonde, J., Bernlohr, D. \& Banaszak, L. (1994). FASEB J. 8, 12401247.

Langer, G., Cohen, S., Lamzin, V. \& Perrakis, A. (2008). Nat. Protoc. 3, 1171-1179.
Liu, W., Ahlert, J., Gao, Q., Wendt-Pienkowski, E., Shen, B. \& Thorson, J. S. (2003). Proc. Natl Acad. Sci. USA, 100, 11959-11963. Ma, G., Tran, H., Low, Z., Candra, H., Pang, L., Cheang, Q., Fang, M. \& Liang, Z. (2021). J. Am. Chem. Soc. 143, 11500-11509.

Morin, A., Eisenbraun, B., Key, J., Sanschagrin, P., Timony, M., Ottaviano, M. \& Sliz, P. (2013). eLife, 2, e01456.

Myers, A., Fraley, M., Tom, N., Cohen, S. \& Madar, D. (1995). Chem. Biol. 2, 33-43.

Nicolaou, K., Hummel, C., Nakada, M., Shibayama, K., Pitsinos, E., Saimoto, H., Mizuno, Y., Baldenius, K. \& Smith, A. (1993). J. Am. Chem. Soc. 115, 7625-7635.

Nicolaou, K., Smith, A., Wendeborn, S. \& Hwang, C. (1991). J. Am. Chem. Soc. 113, 3106-3114.

Pape, T. \& Schneider, T. R. (2004). J. Appl. Cryst. 37, 843-844.

Savitsky, P., Bray, J., Cooper, C. D. O., Marsden, B. D., Mahajan, P., Burgess-Brown, N. A. \& Gileadi, O. (2010). J. Struct. Biol. 172, 313.

Shah, N. (2017). Curr. Drug Targets, 18, 257-270.

Shair, M., Yoon, T., Mosny, K., Chou, T. \& Danishefsky, S. (1996). J. Am. Chem. Soc. 118, 9509-9525.

Sheldrick, G. M. (2010). Acta Cryst. D66, 479-485.

Smanski, M., Yu, Z., Casper, J., Lin, S., Peterson, R., Chen, Y., WendtPienkowski, E., Rajski, S. \& Shen, B. (2011). Proc. Natl Acad. Sci. USA, 108, 13498-13503.

Sugiura, Y., Shiraki, T., Konishi, M. \& Oki, T. (1990). Proc. Natl Acad. Sci. USA, 87, 3831-3835.

Thorson, J., Sievers, E., Ahlert, J., Shepard, E., Whitwam, R., Onwueme, K. \& Ruppen, M. (2000). Curr. Pharm. Des. 6, 18411879.

Van Lanen, S. \& Shen, B. (2008). Curr. Top. Med. Chem. 8, 448-459.

Yennamalli, R., Arangarasan, R., Bryden, A., Gleicher, M. \& Phillips, G. N. Jr (2014). J. Appl. Cryst. 47, 1153-1157. 